\title{
Artificial Intelligence in POCUS: The Vanguard of Technology in COVID-19 Pandemic
}

\author{
Srinath Damodaran ${ }^{1}$ Arjun Alva² Srinath Kumar ${ }^{3}$

\begin{abstract}
${ }^{1}$ Department of Anaesthesia and Intensive Care, Narayana Institute of Cardiac Sciences, Narayana Health City, Bangalore, Karnataka, India.

2Department of Critical Care, Narayana Institute of Cardiac Sciences, Narayana Health City, Bangalore, Karnataka, India

${ }^{3}$ Department of Emergency Medicine, Narayana Institute of Cardiac Sciences, Narayana Health City, Bangalore, Karnataka, India.

${ }^{4}$ Department of Anaesthesia and Intensive Care, Narayana Institute of Cardiac Sciences, Narayana Health City, Bangalore, Karnataka, India.
\end{abstract}

Muralidhar Kanchi ${ }^{4}$

\begin{abstract}
Address for correspondence Muralidhar Kanchi, MD, FIACTA, FICA, MBA, FASE, Department of Anaesthesia and Intensive Care, Narayana Institute of Cardiac Sciences, Narayana Health City, Bangalore, Karnataka, 560099, India

(e-mail: muralidhar.kanchi.dr@nhhospitals.org).
\end{abstract}

J Card Crit Care:2020;4:116-120
Abstract
Keywords
- lung ultrasound
- B line
- artificial intelligence
- COVID-19
- algorithms

The creation of intelligent software or system, machine learning, and deep learning technologies are the integral components of artificial intelligence. Point-of-care ultrasound involves the bedside use of ultrasound to answer specific diagnostic questions and to assess real-time physiologic responses to treatment. This article provides insight into the pearls and pitfalls of artificial intelligence in point-of-care ultrasound for the coronavirus disease 2019 pandemic.

\section{Introduction}

The recent pneumonia outbreak spreading from Wuhan, China, in December 2019, is caused by the 2019 novel coronavirus (2019-nCoV) infection, defined as new coronavirus disease 2019 (COVID-19). ${ }^{1}$ Since COVID-19 is primarily a respiratory disease, the expectation would be that lung imaging would be essential for the diagnosis. ${ }^{2}$ Ultrasound is rapidly becoming the imaging technique of choice in the assessment of COVID-19 cases. Ultrasound imaging of the lung and associated tissues may help inform clinical decision making for patients with COVID-19 and management of their associated respiratory failure and lung injury. Moreover, myocardial injury may be present in more than a quarter of patients with critical disease. ${ }^{3}$ Thus, the rapid bedside assessment of the heart, chest, and vessels by point-of-care ultrasound (POCUS) has propelled this tool to the frontlines of the fight against the COVID-19 pandemic. POCUS is usually conducted by the treating healthcare provider to obtain real-time information for diagnosis and management decisions. In addition to point-of-care use, POCUS has major utility for management of COVID-19 due to its portability, safety, repeatability, absence of radiation, and low cost compared with computed tomography (CT) scan. With the arrival of artificial intelligence (AI) in medicine practice especially in radiology field, diagnosis can be obtained rapidly by treating physicians at the bedside.

\section{COVID-19 and Lung POCUS}

COVID-19 pneumonia is associated with the ultrasound findings of pleural line irregularities and B line artifacts, which are both caused by interstitial thickening and inflammation and increase in number with severity. ${ }^{4}$ The $B$ line is an artifact with seven criteria (the first three constant and the four last quite constant): comet-tail, arising from the pleural line, moving with lung sliding, well defined, long, hyperechoic, and erasing A lines. ${ }^{5}$ In the later stage of disease, lung ultrasound shows consolidation patterns with para-pneumonic effusions. Since COVID-19 affects subpleural region commonly, ultrasound detects published online

November 6, 2020
DOI https://doi.org/ $10.1055 / \mathrm{s}-0040-1718983$ ISSN 2457-0206.
(C) 2020. Official Publication of The Simulation Society (TSS), accredited by International Society of Cardiovascular Ultrasound (ISCU).

This is an open access article published by Thieme under the terms of the Creative Commons Attribution-NonDerivative-NonCommercial-License, permitting copying and reproduction so long as the original work is given appropriate credit. Contents may not be used for commercial purposes, or adapted, remixed, transformed or built upon. (https://creativecommons.org/licenses/by-nc-nd/4.0/)

Thieme Medical and Scientific Publishers Pvt. Ltd., A-12, 2nd Floor, Sector 2, Noida-201301 UP, India 
the lesion clearly. ${ }^{6}$ Moreover, POCUS helps in the rapid assessment of the severity of severe acute respiratory syndrome coronavirus 2 pneumonia/acute respiratory distress syndrome (ARDS) at presentation and triaging, to track the evolution of disease, to monitor lung recruitment maneuvers, to guide response to prone position, the management of extracorporeal membrane therapy, and for making decisions related to weaning the patient form ventilator

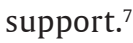

\section{COVID-19 and Cardiac POCUS}

In one cohort of 191 patients from Wuhan, China, any comorbidity was present in $48 \%$ (67\% of nonsurvivors), hypertension (HTN) was present in 30\% ( $48 \%$ of nonsurvivors), diabetes mellitus (DM) in 19\% (31\% of nonsurvivors), and cardiovascular disease (CVD) in $8 \%$ (13\% of nonsurvivors). ${ }^{8}$ Data from the National Health Commission of China demonstrated that 35\% of patients diagnosed with COVID-19 had HTN and 17\% had coronary heart disease. ${ }^{9}$ Potential explanations include CVD being more prevalent in those with advancing age, a functionally impaired immune system, elevated levels of angiotensin converting enzyme 2, or a predisposition to COVID-19 for those with CVD. ${ }^{3}$ Myocardial injury, evidenced by elevated cardiac biomarkers, is common in COVID-19 disease. The possible causes mentioned include cytokine storm, myocarditis, or stress cardiomyopathy. ${ }^{8,10}$ POCUS can help to quickly identify the circulatory status of novel coronavirus patients and guide hemodynamic management. Some authors suggested to measure the diameter of inferior vena cava (IVC) and its collapsibility index, cardiac output (CO), velocity-time integral of the left ventricular outflow (LVOT VTI) during continuous and dynamic evaluation of patients' volume state and fluid responsiveness, left ventricular systolic function, and left ventricular output effect. ${ }^{11}$

\section{Novices in POCUS}

During COVID-19 pandemic, experienced ultrasonographer for imaging all COVID-19 patients may not be possible. Hence, ultrasound machine with AI technology may be essential in some hospitals. Many countries are witnessing the shortage of ultrasound-trained physicians and sonographers. So, it may be necessary to utilize nontrained or less experienced ultrasonographers and physicians to rapidly assess thousands of critical and noncritical COVID-19 cases. Thus, rapid training of such candidates is necessary in short period of time without any compromise on the quality of care. In this kind of scenario, a technology is required to obtain, optimize, and diagnose a condition with foolproof even with less experienced ultrasonographers. The system should have good image quality along with accurate interpretation of abnormal findings and rapid measurement of various parameters of organs in a short span of time. Moreover, there is fewer teaching staff to train the novice ultrasonographers in current situation which mandates a system or software that requires easy teaching. Here, comes the role of AI in POCUS!

\section{Artificial Intelligence in POCUS}

$\mathrm{AI}$ is a machine's ability to mimic human intelligence. In practice, it is a segment of computer science that involves designing computer applications to perform tasks that typically have required human intelligence such as visual perception, speech recognition, and decision making. In a recent proposal aimed at regulating AI software in medical devices, the U.S. Food and Drug Administration (FDA) states that "Artificial intelligence-based technologies have the potential to transform healthcare by deriving new and important insights from the vast amount of data generated during the delivery of healthcare every day." ${ }^{2}$ Instead of instructing the computer to evaluate a given condition, or to perform a specific task according to detailed programmed instructions, AI algorithms learn from exposure to numerous similar conditions previously, which is very useful in dealing with COVID-19 patients. AI is particularly useful in radiology field, as already many AI-based algorithms are implemented in X-ray, CT, magnetic resonance imaging (MRI), and positron emission tomography scans. In the same line, some vendors are providing automatic calculation of B line score, LVOT VTI, and IVC collapsibility index in POCUS. Recently, FDA accelerated the process to provide rapid clearance for AI-based medical imaging technologies to help clinicians in COVID-19 pandemic.

At this institution, an ultrasound machine with $\mathrm{AI}$ (Venue Go, GE Healthcare, Wisconsin, United States) has been installed for rapid identification of B lines, IVC collapsibility index, and auto-VTI exclusively dedicated for imaging COVID-19 patients (-Figs. 1 and 2). The AI model provided by one vendor is shown in - Fig. 3. Introduction of AI in POCUS decreases the keystrokes-a welcome benefit in COVID-19 pandemic. In addition to the AI, complete touch screen technology of this machine is advantageous in current situation, where disinfecting the machine is easy. Disinfection of the machine should be done as per manufacturer's recommendations. ${ }^{13}$

Calculation of $B$ line scoring using ultrasound machine with $\mathrm{AI}$ is a simple process. The curvilinear probe should be placed on each lung zone, as instructed by machine. This should be followed by activating the auto-B key. The number of $B$ lines in a particular zone is exhibited on the screen as a numerical value by the automated software ( - Fig. 4 ).

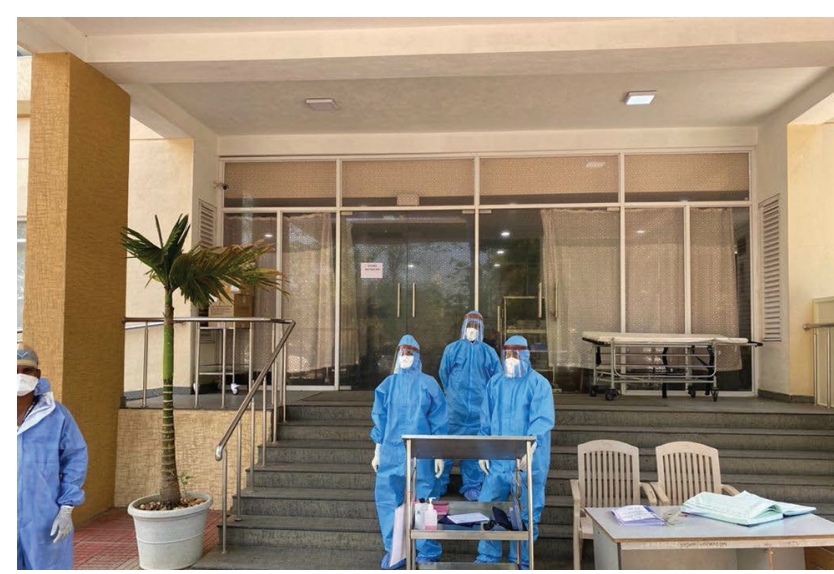

Fig. 1 Coronavirus disease 2019 block in Narayana Health City, Bangalore, Karnataka, India. 


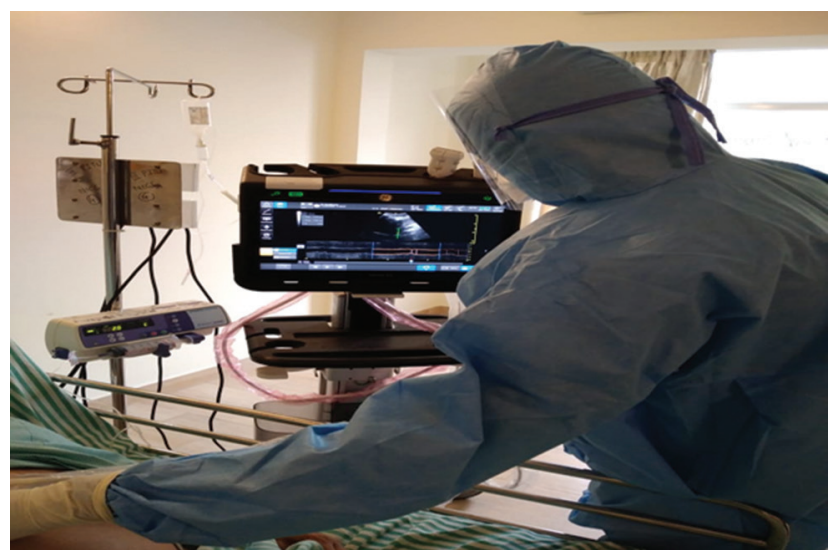

Fig. 2 Point-of-care ultrasound using ultrasound machine with artificial intelligence in a coronavirus disease 2019 suspected patient while waiting for results of reverse transcriptase polymerase chain reaction testing.

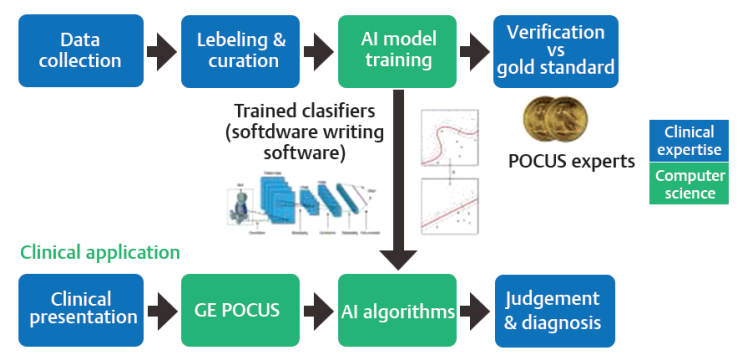

Fig. 3 Artificial intelligence pathway (courtesy: GE Healthcare, Wisconsin, United States). POCU, point-of-care ultrasound.

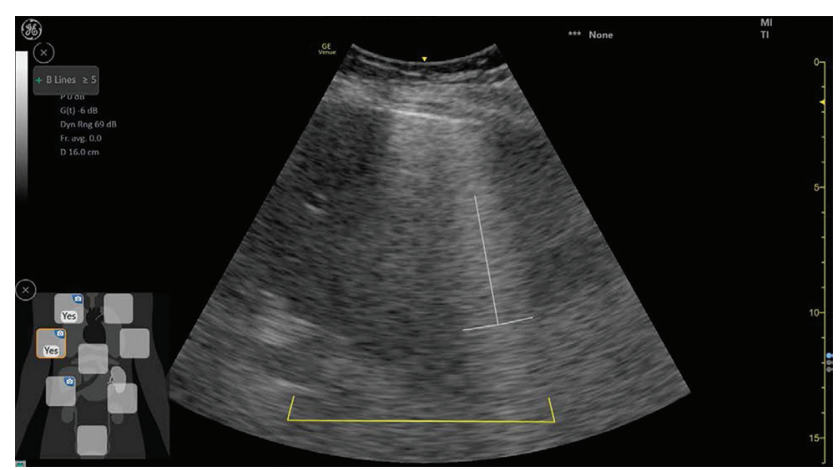

Fig. 4 B line scoring using ultrasound machine with artificial intelligence in a 72-year-old female coronavirus disease 2019 suspected patient ( $\mathrm{B}$ lines $>5$ ).

Same technique should be followed for all lung zones in both lungs. The machine has additional benefit of reviewing all lung fields together to arrive at the final impression. Buonsenso et al suggested ultrasound could be possible replacement of stethoscope in COVID-19 patients because of more contagious nature of the organism..$^{14}$ Incorporation of $\mathrm{AI}$ in ultrasound/POCUS further improves the diagnostic accuracy with more time saving for both ultrasonographer and patient, which is very much needed in COVID-19. To increase the utility of ultrasound by nontraditional expert, vendors need to make ultrasound systems easier and more intuitive to use without compromising on scan quality. AI technology and deep learning techniques are there to address this challenge. Thus, AI-based POCUS performed by nontraditional experts can be a surrogate for expert POCUS when rapid assessment is needed.

Brusasco et al compared automatic quantification of B lines by AI with semiquantitative scores in the measurement of extravascular lung water as determined by standard thermodilution..$^{15}$ Author found that that computer-aided measurement of the percentage of pleural line affected with B line artifacts can provide a reliable operator independent assessment of extravascular lung water in ARDS, which seems to be comparable or superior to previously described scores. Further, author mentioned that this method is faster than conventional method of extra lung water estimation. This study is important in current situation of COVID-19 pandemic where mortality is common due to ARDS. Excessive fluid administration in such patients can be catastrophic because of extra lung water accumulation, which can be rapidly assessed by auto-B lines scoring. Increase in $B$ line score predicts fluid accumulation in lung.

Peng et al reported that the ultrasound can be able to detect lung changes in early stages of COVID-19; they also highlighted the importance of POCUS as a screening tool in fields to detect, trace, and isolate COVID-19 cases while awaiting for reverse transcriptase polymerase chain reaction results. ${ }^{4}$ Automated computer-aided measurement of aeration further simplifies the process, in which B line-based scoring system, along with oxygen requirement status, can be used for categorizing the patients into mild, moderate, severe, and critical for rapid triaging in emergency room. At this institution, we have developed a triaging protocol for COVID-19 using lung ultrasound as shown in - Fig. 5.

Evidences showed that COVID-19 can affect heart directly or indirectly through cytokine storm causing decrease in left ventricular ejection fraction and CO; auto-VTI is helpful in calculating CO rapidly. ${ }^{3,8}$ Calculation of VTI and CO using ultrasound machine with $\mathrm{AI}$ is a single-step process by placing the probe in apical five-chamber view and pressing the auto-VTI key (-Fig. 6). Moreover, this software also provides VTI treading over a period of time to see the effect of treatment. Previously, Shaikh et al evaluated the feasibility of using $\mathrm{AI}$ in POCUS assessments of $\mathrm{CO}$ and to compare the accuracy of automated versus manual measurements of left ventricular outflow tract diameter and LVOT VTI. Shaikh et al also reported that automatic VTI measurement by AI is feasible and allows for quick and accurate measurement of $\mathrm{CO}$ in novice operators. ${ }^{16}$ Further, they suggested $\mathrm{AI}$ in POCUS diagnostics may limit expertise dependence and improve accuracy in nonexperienced users.

Similarly, auto-intelligence can be used to calculate IVC collapsibility index to assess the volume status and diagnosing the cause for decline in hemodynamic status. Calculation of IVC collapsibility index is again a single-step process by using ultrasound machine with AI. This is obtained by placing the probe in subcoastal view and touching the auto-IVC key ( - Fig. 7). Ultrasound screen will automatically display the 


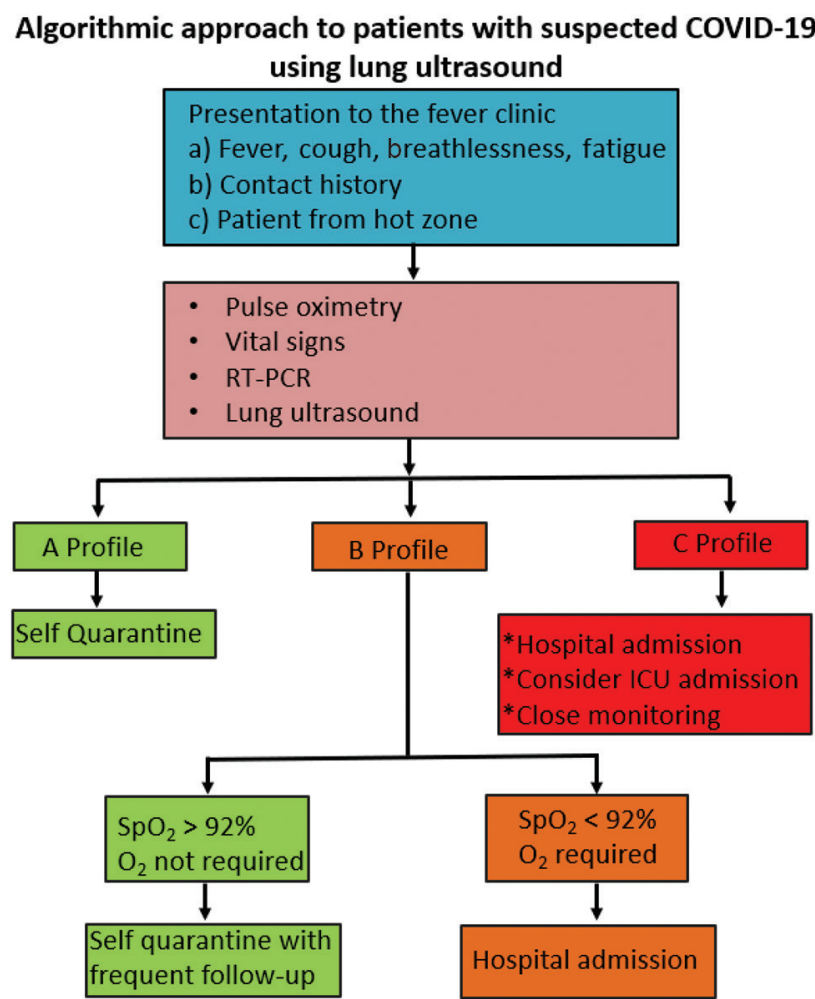

Fig. 5 Triaging protocol for coronavirus disease 2019 (COVID-19) using lung ultrasound in Narayana Health City, Bangalore, Karnataka, India. RT-PCR, reverse transcription polymerase chain reaction; ICU, intensive care unit; $\mathrm{SpO}_{2}$, blood oxygen saturation level.

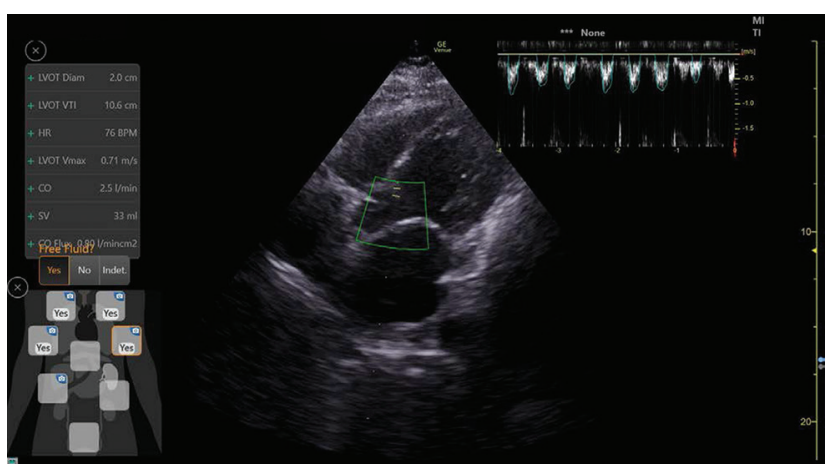

Fig. 6 Auto-velocity time integral and cardiac output using ultrasound machine with artificial intelligence in a 72-year-old female coronavirus disease 2019 suspected patient (cardiac output: $2.5 \mathrm{~L}$ ) min, left ventricular velocity time integral: $10.6 \mathrm{~cm}$ ).

values. In one internal study conducted by GE Healthcare, the IVC measures were equivalent to an expert user's ability $90 \%$ of the time for minimal diameters and $97 \%$ for maximal diameters. ${ }^{17}$

In the current scenario of COVID-19 pandemic, healthcare workers are working round the clock in hospitals. This extreme burden on healthcare system leads to human errors. Extreme work stress can cause physical and mental fatigue hampering the analytical thinking process. Computer-aided decision making could address these human errors caused by fatigue and tiredness.

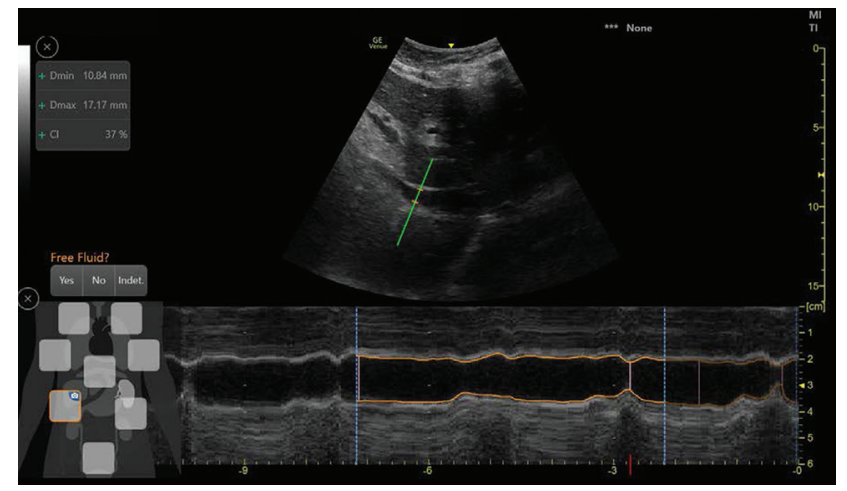

Fig. 7 Auto-inferior vena cava collapsibility index calculation using ultrasound machine with artificial intelligence in a 72-year-old female coronavirus disease 2019 suspected patient (inferior vena cava maximum diameter: $1.71 \mathrm{~cm}$, minimum diameter: $1.08 \mathrm{~cm}$, collapsibility index: $37 \%)$.

\section{Pitfalls}

AI-integrated POCUS machines can be expensive. Ultrasound may not able to identify deep lung lesions, chronicity of lesions, and super added bacterial infections, which may require other diagnostic modalities such as $\mathrm{CT}$ scan and MRI. ${ }^{18}$ Though some argue that AI would decrease the requirement of expert doctor's role and machine dependent, it is much more needed in this pandemic situation in countries such as India, where less health workers are available. Moreover, it is not going to eliminate physicians. Symbiotic relation will continue to exist between physician and machines.

\section{Future Developments}

Current pandemic provides enough vision on how to tackle subsequent similar situations. We have to prime the AI with sufficient information on cases for developing future algorithms to ease the job. Giving more and more data about the disease features to AI improves its accuracy. New algorithms should be developed using AI technology for rapid quantification of effusion, right heart functions, valvular disease, and pulmonary HTN. Designing software with various emergency protocols based on the data available from hemodynamic monitor and diagnostic modalities will provide more conclusive evidence for final diagnosis rapidly. Newer technologies such as elastography, speckle tracking echocardiography, and contrast-enhanced ultrasound that are being used in intensive care unit patients should also be included. As the technology advances by each day, time is not so far away to achieve these necessities. Last, combination of robotic technology with AI technology in POCUS would do miracles in near future, which prevents healthcare workers from infection of contagious disease such as COVID-19.

\section{Conclusion}

AI in POCUS will become part of our working environment in every hospital in the not too distant future, with all 
stakeholders working together to provide a safe, effective service for patients especially affected by COVID-19 like diseases.

\section{Source of Support}

Nil.

\section{Disclosure of Conflict of Interest}

None.

\section{References}

1 Zhu N, Zhang D, Wang W, et al; China Novel Coronavirus Investigating and Research Team. A novel coronavirus from patients with pneumonia in China, 2019. N Engl J Med 2020;382(8):727-733

2 Kanne JP, Chest CT. Chest CT findings in 2019 novel coronavirus (2019-nCoV) infections from Wuhan, China: key points for the radiologist. Radiology 2020;295(1):16-17

3 Clerkin KJ, Fried JA, Raikhelkar J, et al. Coronavirus disease 2019 (COVID-19) and cardiovascular disease. Circulation 2020;141(20):1648-1655

4 Peng QY, Wang XT, Zhang LN; Chinese Critical Care Ultrasound Study Group (CCUSG). Findings of lung ultrasonography of novel corona virus pneumonia during the 2019-2020 epidemic. Intensive Care Med 2020;46(5):849-850

5 Lichtenstein D, Lung ultrasound in the critically ill. In: 2004 Yearbook of Intensive Care and Emergency Medicine. Berlin, Germany: Springer-Verlag; 2004 625-644

6 Huang, Y, Wang S, Liu Y et al. A preliminary study on the ultrasonic manifestations of peripulmonary lesions of non-critical novel coronavirus pneumonia (COVID-19). SSRN 2020. Epub 28 February

7 Soldati G, Smargiassi A, Inchingolo R, et al. Is there a role for lung ultrasound during the COVID-19 pandemic? J Ultrasound Med 2020;39(7):1459-1462
8 Zhou F, Yu T, Du R, et al. Clinical course and risk factors for mortality of adult inpatients with COVID-19 in Wuhan, China: a retrospective cohort study. Lancet 2020;395(10229):1054-1062

9 Zheng Y-Y, Ma Y-T, Zhang J-Y, Xie X. COVID-19 and the cardiovascular system. Nat Rev Cardiol 2020;17(5):259-260

10 Oudit GY, Kassiri Z, Jiang C, et al. SARS-coronavirus modulation of myocardial ACE2 expression and inflammation in patients with SARS. Eur J Clin Invest 2009;39(7):618-625

11 Peng Q-Y, Wang X-T, Zhang L-N; Chinese Critical Care Ultrasound Study Group (CCUSG). Using echocardiography to guide the treatment of novel coronavirus pneumonia. Crit Care 2020;24(1):143

12 Proposed Regulatory Framework for Modifications to Artificial Intelligence/Machine Learning (AI/ML)-Based Software as a Medical Device (SaMD). https://www.fda.gov/media/122535/ downloads. Accessed Aug 14, 2020

13 https://www.gehealthcare.com/products/ultrasound/ultrasound-transducers\#generalcare. Accessed Aug 14, 2020

14 Buonsenso D, Pata D, Chiaretti A. COVID-19 outbreak: less stethoscope,moreultrasound.LancetRespirMed2020;8(5):e2710.1016/ S2213-2600(20)30120-X

15 Brusasco C, Santori G, Bruzzo E, et al. Quantitative lung ultrasonography: a putative new algorithm for automatic detection and quantification of B-lines. Crit Care 2019;23(1):288

16 Shaikh F, Awan O, Friedman O, Qadir THN, Barjaktarevic I. Measuring the accuracy of cardiac output using POCUS: the introduction of artificial intelligence into routine care. Am J Respir Crit Care Med 2020;201:A1775

17 Venue Go R2 Technical Product Claims Document (DOC2199650)

18 Cheung JCH, Lam KN. POCUS in COVID-19: pearls and pitfalls. Lancet Respir Med 2020;8(5):e3410.1016/ S22132600 (20)301661 